\title{
Wear Mechanisms in Functionally Graded Aluminium Matrix Composites: Effect of the Lubrication by an Aqueous Solution
}

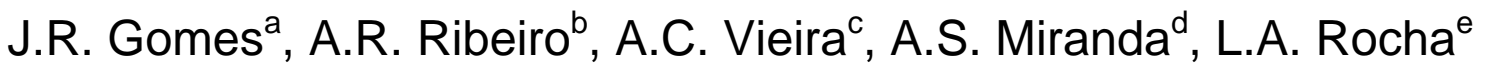 \\ Dept. of Mechanical Engineering, CIICS, University of Minho, 4800-058 Guimarães, Portugal \\ ajgomes@dem.uminho.pt, banaribeiro@engmateriais.eng.uminho.pt, \\ ccatarina.vieira@engmateriais.eng.uminho.pt, ${ }^{\mathrm{d}}$ asm@dem.uminho.pt, ${ }^{\mathrm{e}}$ Irocha@dem.uminho.pt
}

Keywords: FGMs, aluminum matrix composites, wear, friction, tribocorrosion.

\begin{abstract}
Functionally graded aluminium matrix composites reinforced with SiC particles are attractive materials for a broad range of engineering applications in the automotive, aircraft, sports, military and aerospace industries, whenever a superior combination of surface and bulk mechanical properties are required. In general, these materials are developed for the production of high wear resistant components. Also, often this kind of mechanical part operates in the presence of aggressive environments, such as marine atmospheres. In this work, aluminium composites with functionally graded properties, obtained by centrifugal cast, are characterised by reciprocating pin-on-plate sliding wear tests against nodular cast iron. Three different volume fractions of SiC reinforcing particles in each functionally graded material were considered. Sliding experiments were performed with and without the presence of a lubricant ( $3 \% \mathrm{NaCl}$ aqueous solution). All tests were carried out at room temperature, under a normal load of $10 \mathrm{~N}$ and constant frequency $(1 \mathrm{~Hz})$ and stroke $(6 \mathrm{~mm})$. In the case of the lubricated tests, electrochemical parameters (corrosion potential) were monitored during sliding. The worn surfaces as well as the wear debris were characterised by SEM/EDS.

Friction values were in the order of 0.42 for unlubricated conditions, but varied between 0.22 and 0.37 when the aqueous solution was present. Relatively high wear rates (over $1 \times 10^{-6} \mathrm{gm}^{-1}$ ) were obtained in both unlubricated and lubricated sliding. The volume fraction of SiC particles exerted a net effect on the tribological response of the composites, although conditioned by the presence or absence of the aqueous solution. A deleterious effect of the chloride aqueous solution on the degradation of the matrix, leading to the pull-out of $\mathrm{SiC}$ particles induced by the dissolution of the matrix/particle interface was evidenced. Observation of the worn surface morphology indicated that the presence of the lubricant modifies the protective action promoted by the combined effect of the presence of reinforcing particles as load bearing elements and the formation of adherent iron-rich tribolayers. The evolution of the corrosion potential during the sliding action is in accordance to the degradation mechanisms proposed for these systems.
\end{abstract}

\section{Introduction}

The research on functionally graded materials (FGMs) is motivated by the need for properties that are unavailable in any single material and the need for graded properties to offset adverse effects of discontinuities for layered materials [1]. The application of this concept to metal matrix composites leads to the development of materials or components designed with the purpose of being selectively reinforced only at regions requiring increased modulus, strength and/or wear resistance [2]. This kind of composites are a relatively new class of engineered materials that are characterised by a continuous or gradual variation in composition and in the microstructure across the material thickness; these variations cause a change in the properties of the material. Thus, in FGMs, the constituents vary in some direction enabling these materials to provide unique performance. Usually, these composites are provided with ceramic particles on the side where high wear/high temperature resistance is required and tough metals in the side where good mechanical properties and high thermal conductivity are needed. Among the group of hard ceramic particles considered for inclusion in 
aluminium matrix composites, SiC particles have been found to have an excellent compatibility with the aluminium matrix and can be obtained at low cost. Other attractive properties of these composites include their excellent wear resistance and their ease processing [3].

Considerable progress has been made in recent years in the development of the processing techniques of FGMs. These techniques may be grouped into: liquid phase techniques, solid phase techniques and vapour phase techniques. Centrifugal casting can be considered as one of the most cost and technical effective methods to produce functionally graded materials when Al-based alloys are mixed with SiC particles. The main fields of application of these composites are aircraft, automotive, sports, military and aerospace industry [4]. These kind of applications requires high wear resistance that FGM can give. Therefore, the characterisation of prevailing wear mechanisms that can occur in contact situations involving functionally graded aluminium matrix composites has recently drawn more attention because provide a criterion for material selection, engineering design, failure analysis and wear life estimation [5].

Materials used for tribological applications should be able to support a load without undue distortion, deformation or fracture, and should have low wear almost always combined with low friction coefficient under sliding contact. The friction and wear behaviour of the composites is known to be dependent on the microstructural characteristics of the matrix and also on the type, shape, size and volume fraction of the reinforcing particles. Traditionally, non-corrosive liquid lubricants externally added to the system play an important role, reducing the friction coefficient and the wear damage of the mating surfaces. However if the lubricant is corrosive (like aqueous solutions of $\mathrm{NaCl}$ ), important changes take place in the tribosystem as new surface degradation mechanisms occur.

A high number of studies are reported in literature regarding the tribological behaviour of homogeneous metal matrix composites, but the information about the tribological response of these materials when prepared as FGMs is scarce and their tribological performance in the presence of corrosive environments is still missing. In this work, the wear mechanisms in functionally graded aluminium matrix composites are investigated by reciprocating pin-on-plate sliding wear tests. The experiments were performed without lubrication and in the presence of an aqueous solution of 3\% $\mathrm{NaCl}$ to simulate a corrosive environment. A comparative analysis is established between unlubricated and lubricated tests in order to understand the tribocorrosion behaviour of aluminium matrix composites.

\section{Experimental}

The fabrication process of the functionally graded aluminium matrix composites was carried out in a high frequency induction centrifugal casting furnace as described by Gomes el al. [1,2,4]. Cylindrical samples were produced with the SiC content varying along their geometric axis, from which discs were machined. Three cross-sections of the cylindrical samples, identified in Table 1 as A, B and C, placed respectively at the outer edge (considering the positive direction of the centrifugal force), 5 $\mathrm{mm}$ and $20 \mathrm{~mm}$ from the outer edge, were considered as plates for the tribological tests. Table 1 presents details of the chemical composition, particle fraction area and hardness of the composite plates and of the cast iron pins.

Unlubricated and lubricated reciprocating sliding wear tests were performed in an adapted pin-on-disc tribometer (Plint, TE67). The experiments were conducted at room temperature under a normal applied load of $10 \mathrm{~N}$, constant frequency $(1 \mathrm{~Hz})$, stroke $(6 \mathrm{~mm})$ and total sliding distance $(120 \mathrm{~m})$. A bending type force transducer was used to assess the friction coefficient and the wear rate was measured by the weight loss method.

Prior to testing, the surfaces of both cast iron pins and composite plates were polished, ultrasonically cleaned, dried and finally weighted. For unlubricated tests, structural studies by scanning electron microscopy (SEM) and energy dispersive X-ray (EDS) analysis were used to characterise the plate and pin worn surfaces as well as the loose wear debris. In the case of lubricated 
tests, a solution of $3 \% \mathrm{NaCl}$ was used to simulate the corrosive environment. All experimental parameters were maintained, and a PGZ 100 Voltalab was used for the electrochemical measurements. The contact area was immersed in the solution (electrolyte) and its open circuit potential was measured using a standard three electrode cell. The reference electrode used was calomel, the working electrode was the plate and the counter electrode was a wire of platinum. During the experiment, all the electrochemical parameters such as potential were continuously monitored. Morphological and chemical studies of the plate and pin worn surfaces and the loose wear debris produced during the tests were performed by SEM and EDS analysis. Also, chemical analyses by atomic absorption microscopy of the solutions used as electrolyte were performed in a $G B C 904$ AA spectrometer

Table 1 - Composition, particle fraction area and hardness of composite plates and cast iron pins.

\begin{tabular}{lllll}
\hline Material & Composition (wt\%) & $\begin{array}{l}\text { Particle Fraction } \\
\text { Area (\%) }\end{array}$ & $\begin{array}{l}\text { Hardness } \\
\text { (HV30) }\end{array}$ \\
\hline FGM & A & 8.5-9.5\% Si; 0.45-0.65\% Mg; 0.2\% Ti; 25.8 & 80 \\
composite & B & 0.2\% Fe (max); 0.2\% Cu (max) & 33.4 & 89 \\
& C & & 30.5 & 85 \\
Nodular & 3.3\% C; 2.0\% Si; 0.5\% Mn; 0.08 \% S & & \\
cast iron & (max); 0.2\% P (max) & & \\
\hline
\end{tabular}

A - Edge cross section; $\mathrm{B}-5 \mathrm{~mm}$ from the edge; $\mathrm{C}-20 \mathrm{~mm}$ from the edge.

\section{Results and Discussion}

The variations of friction coefficient $(f)$ and wear rate $\left(\mathrm{W}_{\mathrm{r}}\right)$ of different tested pairs under both unlubricated and lubricated conditions are showed in Fig.1. For unlubricated conditions (Fig.1(a)) the friction coefficient remains almost constant $(f \approx 0.42)$, which demonstrate that the friction coefficient is not significantly affected by the particle fraction area of the composite. This is in accordance with the observations made in a previous work [6] where the same tribocouples were tested in a pin-on-disc test configuration. Also, it can be observed that the increase in the amount of SiC particles results in the decrease of the wear rate for both triboelements. The wear loss of the cast iron pin is always higher than that corresponding to the composite plate, as a consequence of an effective abrasive action of the ceramic reinforcing particles. In conclusion, lower friction and wear values were obtained for both pin and plate when the FGM composite B (33.4 \% of particles) is involved in the contact, thus revealing the best tribological system tested under unlubricated sliding. Similar behaviour was previously observed for continuous pin-on-disc sliding [6], and was explained by the particular ability of the FGM composite B to anchor more stable iron-rich protective tribolayers than the other two composites. In addition, the presence of a higher amount of SiC particles, acting as surface profile modifiers and load-supporting elements at the tribocontact, contributes to a high wear resistance of the material [6]. These reinforcing particles, possessing high hardness, can protect the Al matrix from extreme wear damage. However, as a result of the higher tribological loading associated to the reciprocating sliding contact, the wear rates presented in Fig.1(a), which are in the order of $10^{-6} \mathrm{gm}^{-1}$, are ca. two order of magnitude higher than those obtained in a pin-on-disc test configuration [6]. In fact, in reciprocating sliding contacts, several aspects contribute for an increased instability of the surface conditions. Instead of a unidirectional frictional force, we are in the presence of cyclic frictional loading that promotes fatigue and instability of the anchoring iron-rich tribolayers.

The morphological aspects of both worn surfaces, plate and pin, are presented in Fig. 2(a) and (b), respectively. Considering the plate surface, it is characterised by smooth SiC particles protruding above the matrix, which act as load-supporting elements. Also, delamination marks may be found in the matrix. Adherent iron-rich tribolayers are observed, some of them anchored at the protruding SiC particles. Due to the recripocating nature of the test, anchored tribolayers may be observed at both 


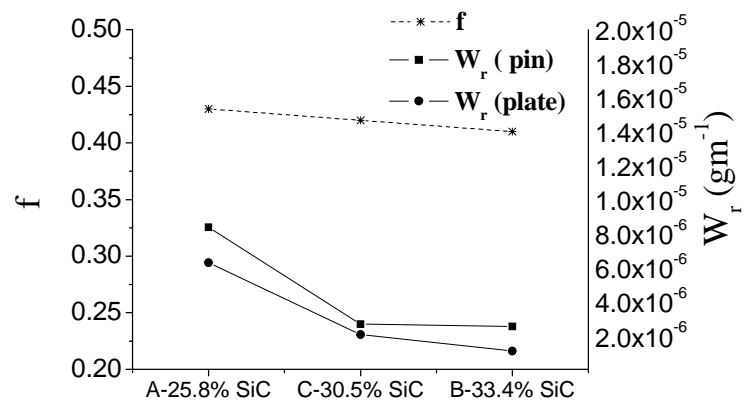

(a)

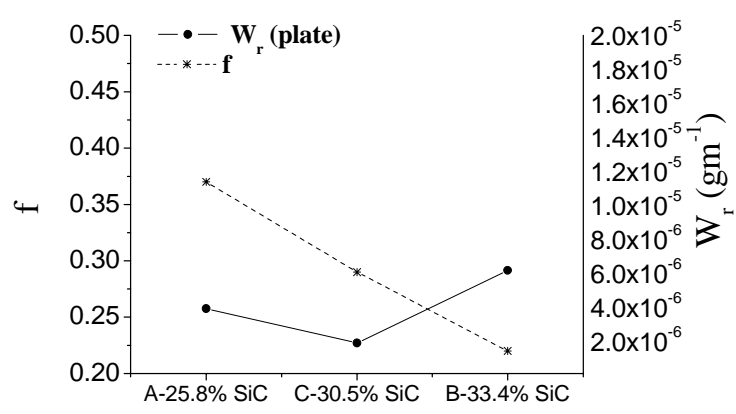

(b)

Fig.1 Relation between friction coefficient $(f)$ and wear rate $\left(W_{r}\right)$ for unlubricated (a) and lubricated (b) tests.

attack sides of the ceramic reinforcing particles (the direction of the sliding movement is horizontal on the micrographs) [6]. Fig. 2(b) is representative of the typical morphology of the worn surface of the cast iron pin after being tested without lubrication. Cavities resulting from the removal of graphite globules are evidenced as well as abrasive grooves aligned with the sliding direction, resulting from abrasion promoted by SiC reinforcing particles from the FGM counterface [3,7]. Another important feature is the presence of $\mathrm{Al}$ based adherent tribolayers transferred from the composite surface.

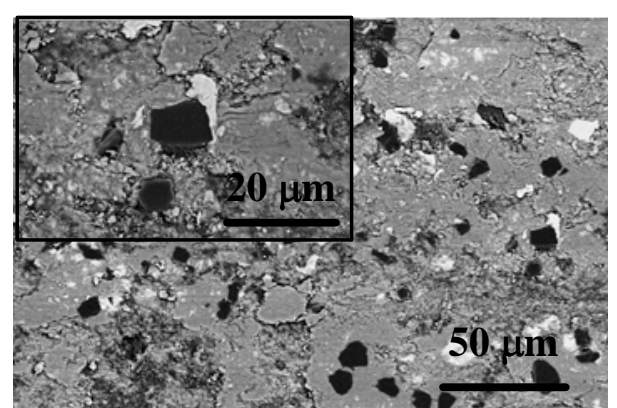

(a)

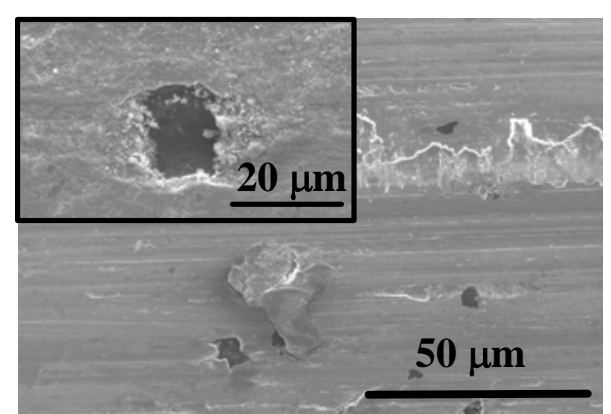

(b)

Fig.2 SEM micrographs of the worn surfaces of FGM composite (backscattered electrons image) (a) and corresponding cast iron pin (b) after sliding under unlubricated conditions.

Under lubrication by aqueous solution, the friction results (Fig.1(b)) are characterised by lower values than for unlubricated sliding, this behaviour being attributed to the lubricant effect given by the solution. However, in this case, the electrolyte has relatively low viscosity, which allows the direct contact between asperities of both mating surfaces, leading the tribosystem to operate under boundary lubrication conditions, i.e. the normal applied load is entirely supported by the contact between asperities. As a consequence the friction values are relatively high $(0.22 \leq \mathrm{f} \leq 0.37)$. At the same time, in lubricated conditions, a remarkable decrease of the friction coefficient with the increase of $\mathrm{SiC}$ content is observed. According to Zhao et al. [8], the amorphous hydrated silica films resulting from the tribochemical reaction between the $\mathrm{SiC}$ and water $\left(\mathrm{SiC}+\mathrm{O}_{2}+\mathrm{H}_{2} \mathrm{O} \rightarrow \mathrm{SiO}_{2}+\mathrm{CO}+\mathrm{H}_{2}\right.$ ) promotes an additional lubricious action, which explains the observed frictional response.

Concerning the wear rate of the plate, it can be seen that the magnitude of the values measured in the lubricated tests is comparable to that from unlubricated experiments. However, composite B (33.4\% SiC) presents a slightly higher $\mathrm{W}_{\mathrm{r}}$, which can be due to the pull-out of SiC particles by a chemically induced fracture mechanism, attributed to a preferential reaction of the aqueous solution with the Al matrix/SiC particle [9]. The composite with intermediate SiC fraction area is the one presenting a lower $\mathrm{W}_{\mathrm{r}}$, which is probably due to the accommodation of Fe-rich oxide layers on the composite surface, as evidenced by EDS analysis. It should be remarked that results concerning the wear rate of the pin, in lubricated tests, are not referred in this work due to the fact of being very 
difficult, from the experimental point-of-view, to control the immersion fraction area of the pin during the sliding motion.

The concentration of released $\mathrm{Al}$, dissolved in the electrolyte after the sliding test, is showed in Fig.3. As it can be seen, the composite containing a lower amount of reinforcing particles is the one giving origin to a greater release of Al. Conversely, an increase in the amount of particles results in a lower quantity of $\mathrm{Al}$ being dissolved in the solution. In fact, two different degradation mechanisms are likely to be occurring in these systems. When the amount of particles is relatively low, the degradation of the material appears to be essentially controlled by the wear/corrosion of the Al-matrix. As the amount of SiC particles increases, the load-carrying capability of the particles becomes more important, resulting in an increased protection of the matrix in what concerns to wear, i.e, the amount of $\mathrm{Al}$ dissolved in the solution decreases. However, the pull-out of the ceramic particles, as a result of the preferential dissolution of the $\mathrm{Al}$ matrix/SiC particle interface above referred, contributes to an additional weight loss of the material, resulting in relatively high $\mathrm{W}_{\mathrm{r}}$ values. The slightly increase in $\mathrm{W}_{\mathrm{r}}$ with the increase of the $\mathrm{SiC}$ fraction area (Fig.1(b)), may be explained by this mechanism.
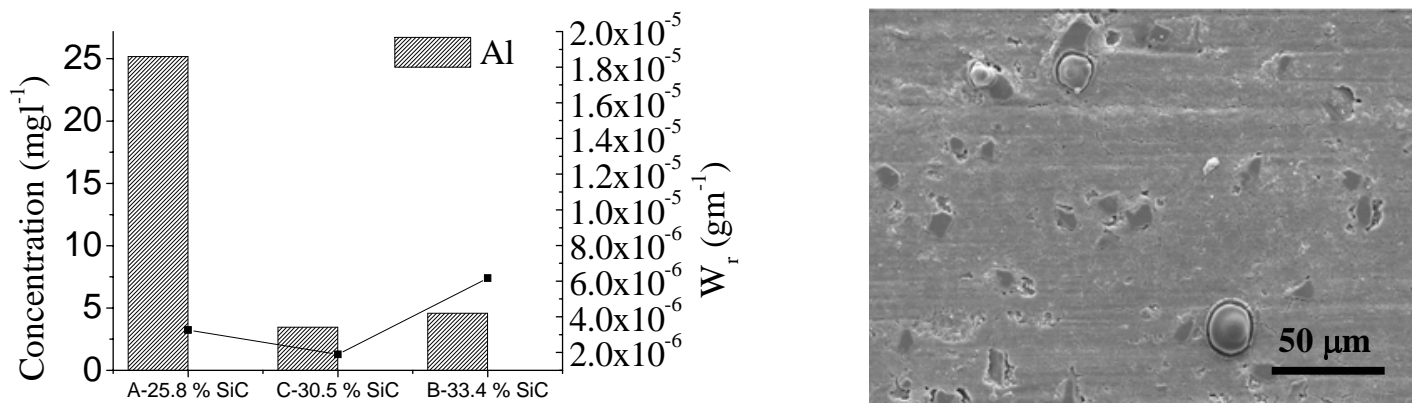

Fig .3 Concentration of released Al, presented in electrochemical solution related with wear rate (a); SEM worn surface micrograph of composite C (b).

Fig.4 displays the evolution of the corrosion potential with time, during sliding. As it can be observed, the potential of the all samples significantly increase when the pin contacts the surface of the plate, due to the formation of the galvanic pair between the two materials. After about 10 minutes the potential of the cast iron/composite pair stabilize in an intermediate value. When sliding starts, the potential suffers a slight increase, which can be attributed to the formation of a film which slightly decreases the corrosion tendency of the system. As discussed above, some transfer of iron occurred to the composite surface, and the film formed due to this transfer is likely to act as a protection to the material, at least from the corrosion point-of-view. As it can be seen in the figure, the potential remains remarkably stable, throughout the sliding period. At the end of sliding, and when the pin is left away from the composite surface, a sudden decrease in potential is observed, and the potential of the plate becomes significantly more active, than before the sliding test. This result clearly shows that the mechanical action causes a destruction of the passive layer which is naturally formed on the Al based alloys. Also, the surface of the material after the sliding tests presents some pores (Fig. 3(b)), which can be approached to pits, formed due to the pull-out of particles during sliding. All these events together may be responsible for the increase in the tendency of the materials for corrosion.

At the end of the sliding test, composite $\mathrm{C}$ have more passive behaviour than the others plates. This behaviour agrees with the fact that this plate has the higher quantity of oxides of Fe tribolayers in comparison with the others plates. Most Fe oxides have an open-circuit corrosion potential of the same order of magnitude of the values obtained in composite $\mathrm{B}$, in the end of sliding test [10]. 


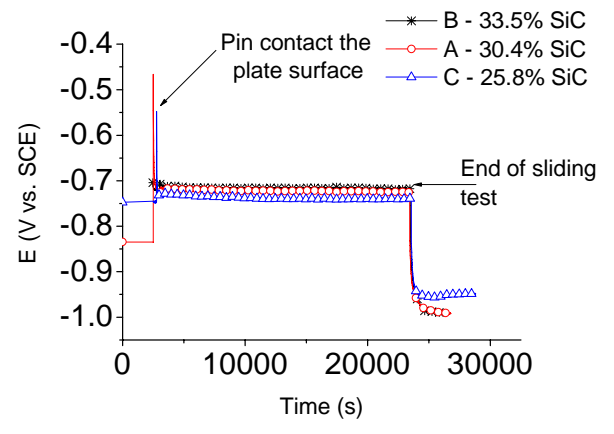

(a)

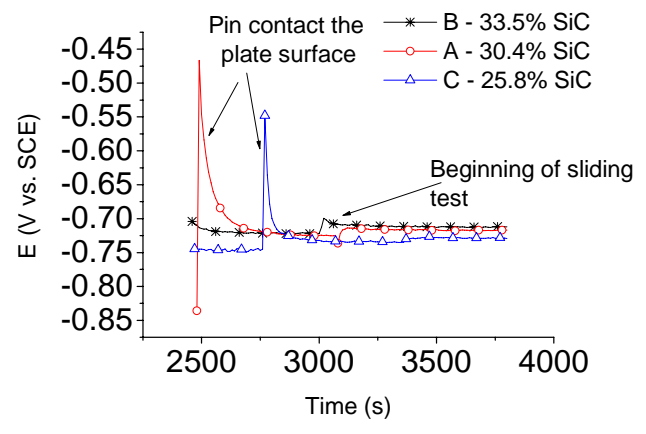

(b)

Fig. 4 (a) Open circuit potential during sliding; (b) Open circuit potential when the pin contact with the surface and the sliding begin.

\section{Conclusions}

For unlubricated sliding the friction coefficient is not significantly affected by $\mathrm{SiC}$ content on the FGM composite ( $\mathrm{f} \approx 0.42$ ). Lower friction and wear values were obtained for the FGM composite with higher amount of reinforcing particles (33.4\%) due to their ability to anchor iron-rich protective tribolayers and load-supporting effect given by SiC particles.

The presence of an aqueous sodium chloride solution does not promote a significant lubricious effect. Nevertheless, it was observed that the friction coefficient decreases with the increase of the amount of $\mathrm{SiC}$ particles, as a result of the products resulting from the reaction of the ceramic particles with the aqueous solution.

The wear rate of the composites is not significantly affected by the presence of the aqueous solution. Together with the corrosion phenomena, two competitive mechanisms were found to determine the weight loss of the material. For the composite with lower amount of reinforcing particles, the removal of material by wear from the Al-based matrix is the main mechanism. However, in the composites with higher amount of particles, pull-out of SiC particles due to the dissolution of the particle/matrix interface becomes dominant.

\section{Acknowledgements}

This work was sponsored by Fundação para a Ciência e Tecnologia (FCT-Portugal) under the Program POCTI (contract POCTI/CTM 46086/2002).

\section{References}

[1] F.M. Xu, S.J. Zhu, J. Zhao, M. Qi, F.G. Wang, S.X. Li, Z.G. Wang: Composites Science and Technology Vol. 64 (2004), p.1795.

[2] J.R. Gomes, A.S. Miranda, L.A. Rocha and R.F. Silva: Key Engineering Materials Vols 230-232 (2002), p. 271.

[3] J.K.M. Kwok, S.C. Lim: Composites Science and Techonology Vol.59 (1999), p.55.

[4] J.R. Gomes, A.S. Miranda, L.A. Rocha, S J. Crnkovic, V. Silva and R.F. Silva: Int J. of Applied Mechanisms and Engineering Vol.7, No. 3 (2002), p. 791.

[5] Mingwu Bai, Qunji Xue and Huifang Guo: Wear Vol. 194 (1996), p. 126.

[6] J.R. Gomes, A.S. Miranda, D. Soares, A.E. Dias, L.A. Rocha, S.J. Crnkovic and R.F. Silva: FGM2000 Vol. 114 (2000), p. 579.

[7] B. Venkataraman and G. Sundararajan: Acta mater Vol 44, No. 2 (1996), p. 451.

[8] L. Fang, Y. Gao, S. Si, Q. Zhou: Wear Vol. 120 (1997), p. 145.

[9] A. Velhinho, J.D. Botas, E. Ariza, J.R. Gomes, L.A. Rocha: Materials2003FGMtribo.

[10] D.A. Jones: Principles and Prevention of Corrosion (Prentice-Hall, Inc. 1996). 